\title{
Disruption of long-term alcohol-related memory reconsolidation: role of $\beta$-adrenoceptors and NMDA receptors
}

\author{
Jelte A. Wouda ${ }^{\dagger}$, Leontien Diergaarde ${ }^{\dagger}$, Danai Riga, Yvar van Mourik, Anton N. M. Schoffelmeer and \\ Taco J. De Vries*
}

Department of Anatomy and Neurosciences, VU University Medical Center, Neuroscience Campus Amsterdam, Amsterdam, Netherlands

\section{Edited by:}

Jacek Debiec, New York University, USA

\section{Reviewed by:}

Yadin Dudai, Weizmann Institute of

Science, Israel

Jacek Debiec, New York University, USA

\section{*Correspondence:}

Taco J. De Vries, Department of Anatomy and Neurosciences,

VU University Medical Center,

Neuroscience Campus Amsterdam

van de Boechorststraat 7, 1081 BT

Amsterdam, Netherlands.

e-mail:tj.devries@vumc.nl

† Jelte A. Wouda and Leontien

Diergaarde have contributed equally.
Disrupting reconsolidation of drug-related memories may be effective in reducing the incidence of relapse. In the current study we examine whether alcohol-related memories are prone to disruption by the $\beta$-adrenergic receptor antagonist propranolol $(10 \mathrm{mg} / \mathrm{kg})$ and the NMDA receptor antagonist MK801 $(0.1 \mathrm{mg} / \mathrm{kg})$ following their reactivation. In operant chambers, male Wistar rats were trained to self-administer a $12 \%$ alcohol solution. After 3 weeks of abstinence, the animals were placed in the self-administration cages and were re-exposed to the alcoholassociated cues for a 20-min retrieval period, immediately followed by a systemic injection of either propranolol, MK801 or saline. Rats were tested for cue-induced alcohol seeking on the following day. Retrieval session, injection and test were repeated on two further occasions at weekly intervals. Both propranolol and MK801 administration upon reactivation did not reduce alcohol seeking after the first reactivation test. However, a significant reduction of alcohol seeking was observed over three post-training tests in propranolol treated animals, and MK801 treated animals showed a strong tendency toward reduced alcohol seeking $(p=0.06)$. Our data indicate that reconsolidation of alcohol-related memories can be disrupted after a long post-training interval and that particularly $\beta$-adrenergic receptors may represent novel targets for pharmacotherapy of alcoholism, in combination with cue-exposure therapies.

Keywords: alcohol, $\beta$-adrenergic receptor, instrumental learning, memory reconsolidation, NMDA receptor, propranolol

\section{INTRODUCTION}

Alcohol consumption is socially accepted in many cultures. The World Health Organization estimates that about 2 billion people regularly drink alcoholic beverages, $4 \%$ of whom have diagnosable alcohol use disorders. The economical, health, and domestic consequences of excessive alcohol use are a burden to society (WHO, 2004). Nevertheless, only few pharmacological treatments of alcohol dependence are available, and their effectiveness is limited (Anton et al., 2006). Therefore, development of more effective treatments is warranted.

In drug addiction, environmental stimuli associated with the effects of self-administered drugs, including alcohol, are powerful sustainers of addictive behaviors and can precipitate relapse after prolonged periods of abstinence in both humans (O'Brien et al., 1998) and laboratory animals (De Vries et al., 2001; Chaudhri et al., 2008). Disrupting drug-associated memories could therefore be an important new strategy for treating alcoholism and other addictive behaviors (Lee et al., 2005; Miller and Marshall, 2005; Diergaarde et al., 2008).

During the last decade, there has been a renewed interest in reconsolidation, a phenomenon involved in retaining reactivated memories. Memory retrieval is believed to induce a transient state during which a memory trace becomes liable to modification, requiring protein synthesis-dependent reconsolidation for the original memory to be maintained. Memory reconsolidation has been studied in a number of species, and localized pharmacological manipulations have identified a variety of brain regions, including basolateral amygdala, hippocampus, and prefrontal cortex, involved in post-reactivation stabilization of memories (Przybyslawski et al., 1999; Nader et al., 2000; Alberini, 2005). The molecular mechanisms underlying reconsolidation involve both $\beta$-adrenergic and glutamatergic signaling pathways (Przybyslawski and Sara, 1997; Przybyslawski et al., 1999; Debiec and LeDoux, 2004). For instance, it has been shown that post-reactivation administration of propranolol, a $\beta$-adrenoceptor antagonist, disrupts reconsolidation of fear-related memories (Przybyslawski et al., 1999; Nader et al., 2000; Debiec and LeDoux, 2004; Alberini, 2005). Similarly, reconsolidation of aversive memory is disrupted by post-retrieval injections of NMDA receptor antagonist MK-801 (Lee et al., 2006b). Collectively, these findings suggest that both $\beta$-adrenergic and glutamatergic signaling pathways are involved in aversive memory reconsolidation processes.

Recent evidence of our and other laboratories indicates that $\beta$-adrenergic signaling is also involved in reconsolidation of appetitive memories. By employing an instrumental sucrose self-administration paradigm, we established that reactivation of relatively old natural reward-related memories renders them susceptible to interference, i.e., propranolol treatment following re-exposure to a sucrose-associated context significantly reduced subsequent sucrose seeking (Diergaarde et al., 2006). It has also been demonstrated that propranolol (Milton et al., 2008b) and MK801 (Lee and Everitt, 2008a) disrupt reconsolidation of associations between sucrose and discrete environmental stimuli. Furthermore, both post-reactivation propranolol, and MK801 administration prior to reactivation 
reduces subsequent cocaine seeking (Milton et al., 2008a,b), which implies that $\beta$-adrenergic and NMDA receptor mediated signaling is also implicated in reconsolidation of cocaine-related associations. Nonetheless, it was only recently shown that protein synthesis and NMDA receptors are also required for reconsolidation of alcoholrelated memories (von der Goltz et al., 2009).

The current study extends on these findings using an operant reinstatement procedure that models cue-induced relapse, to examine whether $\beta$-adrenergic signaling is also involved in reconsolidation of alcohol-related memories. Thus, we compare the effects of propranolol and MK801 administration following memory reactivation.

\section{MATERIALS AND METHODS SUBJECTS}

Male Wistar rats (Harlan CPB, Horst, The Netherlands), weighing 280-300 g upon arrival were used. They were housed in pairs in a temperature and humidity controlled room on a 12-h light/dark cycle (lights on between $7 \mathrm{pm}$ and $7 \mathrm{am}$ ). Food and water were available ad libitum. The experiment started 2 weeks after the animals had arrived and was conducted during the dark phase of the light/dark cycle. Experimental procedures were approved by the Animal Care Committee of the VU University, Amsterdam, The Netherlands.

\section{APPARATUS}

All experiments were conducted in 16 identical rat operant chambers (TSE, Bad Homburg, Germany), which were fitted with a dim red house light and two small holes, one hole in which the rats had to poke in order to obtain a $0.19-\mathrm{ml} 12 \%$ alcohol solution (designated "nose poke hole"), and a hole in which the alcohol was actually delivered (hereby further referred to as "receptacle") located on opposite walls. The nose poke hole contained a photocell to detect responses. Red stimulus lights were located above the nose poke hole and the receptacle, and yellow stimulus lights were located inside both holes. In addition, each chamber was equipped with an empty feeder mechanism which was turned on upon a poke in the nose poke hole, thereby providing an auditory stimulus. The stimuli were used to signal alcohol availability or were paired with alcohol delivery in order to facilitate acquisition of alcohol self-administration (see below). During reactivation and reconsolidation testing, these cues were present to study the effect of propranolol or MK801 on cue-induced alcohol seeking. A computer interfaced to the chambers was used for equipment operation and data collection.

\section{DRUGS}

All drugs were dissolved in sterile saline for intraperitoneal injection $(1 \mathrm{ml} / \mathrm{kg}) .(+/-)$-Propranolol hydrochloride (Sigma) was injected at a dose of $10 \mathrm{mg} / \mathrm{kg}$. (+)-MK-801 hydrogen maleate (Tocris) was injected at a dose of $0.1 \mathrm{mg} / \mathrm{kg}$. These doses of propranolol and MK801 have previously been reported to disrupt reconsolidation of different types of memories (Diergaarde et al., 2006; Sadler et al., 2007).

\section{PROCEDURE}

Experiment 1

Two-bottle procedure. The rats were daily trained to consume alcohol in a two-bottle free-access paradigm. Upon arrival they were habituated to two water bottles on their home cages. Every other day one of the bottles was replaced by a bottle containing a gradually increasing alcohol concentration $(2-12 \% \mathrm{v} / \mathrm{v})$. In 3 weeks the animals reached $12 \% \mathrm{v} / \mathrm{v}$ alcohol and training proceeded to a two-bottle limited-access paradigm. Animals were given access to the $12 \%$ solution for $1 \mathrm{~h}$ daily. After 10 days of limited-access animals that consumed $>0.35 \mathrm{~g} / \mathrm{kg}$ alcohol were selected to enter the operant self-administration phase. Selected animals consumed approximately $0.54 \mathrm{~g} / \mathrm{kg}$ alcohol. Previous work of our laboratory shows that animals with this amount of alcohol intake reliably acquire operant alcohol self-administration and show cue-induced relapse following long-term extinction (De Vries and Schoffelmeer, 2005).

Operant alcohol self-administration. In Figure 2 the experimental setup is depicted. All rats were trained to self-administer a $12 \%$ alcohol solution. On the first day of training they received a single 20-min habituation session, during which 40 non-contingent rewards were delivered and only the house light was illuminated. Nose poking during this session was without any consequences. On the following days the rats were trained to self-administer a $12 \%$ alcohol solution in $1 \mathrm{~h}$ sessions every other day. A session started with the illumination of the house light. Each trial was signaled by illumination of a red stimulus light located above the nose poke hole, which was extinguished when a nose poke was made in this hole. Nose poking resulted in the immediate delivery of a $0.2-\mathrm{ml}$ alcohol solution (US) in the receptacle, signaled by the illumination of a white stimulus light in the receptacle and the sound (click) of the feeder mechanism (compound CS). Responding was reinforced according to a continuous reinforcement (fixed ratio 1, FR1) schedule. After delivery of the reinforcer, the white stimulus light was switched off, and a time-out period of $20 \mathrm{~s}$ commenced until the next trial started. Nose poking behavior during time-out was monitored, but was without consequences. After $1 \mathrm{~h}$ or when 50 reinforcers had been earned, the house light was extinguished and the session ended. In order to facilitate acquisition, the animals received one free sample of alcohol solution in the receptacle upon the start of habituation and the first five training sessions. When FR1 performance levels stabilized, the training schedule proceeded to FR2 and FR3. FR3 training continued until stable peak performance levels were achieved, which indicated that the task was well consolidated. All rats received a total of 19 1-h training sessions.

Alcohol self-administration training was followed by a period of 21 days during which the rats were kept in their home cages in the colony room, and were handled weekly. Rats self-administering less than $0.35 \mathrm{~g} / \mathrm{kg}$ alcohol per session were excluded from analysis.

Reactivation. Following the 3-week abstinence period the rats were subjected to a reactivation session, during which the animals were placed in the self-administration cages for a 20 -min period. During the session the house light and the red stimulus light were presented continuously, the compound CS sound was presented 40 times at variable intervals (VI30, every 25, 30, or 35 s). Thus reactivation consisted of a combination of the context and cues that were present during training, yet, in contrast to the training sessions the nose poke hole as well as the receptacle were covered during reactivation, and no alcohol was delivered. Immediately following reactivation rats were transported to the adjacent room and received an i.p.injection of propranolol $(10 \mathrm{mg} / \mathrm{kg})$ or saline. In 
order to test whether the effect of propranolol depends on memory retrieval, a separate group of animals received identical training and abstinence but was not reactivated. Rats from this no-reactivation experimental group were not placed in the self-administration cages, but instead, were transported to the adjacent room where they received a saline or a propranolol injection.

Retention. The day following memory reactivation, the rats were tested for cue-induced alcohol seeking in a 1-h session. The session was analogous to a FR3 training, with cue presentation upon every third active nose poke, however, alcohol was never delivered. Nose poking was used as an index of alcohol seeking behavior.

In order to test whether repeated propranolol injections in combination with reactivation had an additional detrimental effect on alcohol seeking, retrieval session, injection, and retention test were repeated 7 and 14 days after the first retrieval test.

\section{Experiment 2}

Identical to experiment 1 , alcohol self-administration training was followed by a 21-day period during which the rats were kept in their home cages in the colony room, and were handled weekly. Subsequent reactivation and retention was as described in experiment 1 with the exception that these animals were injected with saline or MK801 (0.1 mg/kg) directly after reactivation.

\section{STATISTICAL ANALYSIS}

SPSS 15.0 for Windows software was used for all statistical analysis and a significance level of $p<0.05$ was selected for all analyses. The reactivation and no-reactivation groups were tested in separate experiments at different points in time, and were therefore subjected to separate statistical analysis. An analysis of variance (ANOVA) in a repeated measures design was used to determine the effects of propranolol and MK801 on alcohol-related memory reconsolidation with retention session (sessions 1-3) as within-subject factor and drug treatment (propranolol/MK801 vs. saline) as between-subject factors. The same analysis was used for the no-reactivation groups. Data were checked for sphericity, and a Huynh-Feldt correction was used if necessary. Active response data failed to meet homogeneity of variance requirements of ANOVA and were $\log _{10}$ transformed prior to statistical analyses. Data are presented as mean \pm SEM active nose poke responses in the figures.

\section{RESULTS}

\section{EXPERIMENT 1: POST-REACTIVATION PROPRANOLOL (10 mg/kg)}

All selected rats learned to respond for alcohol (Figure 1) and demonstrated equivalent levels of active responding during the last 2 days of the 19-day training period [reactivation: $F(1,19)<1$, $p>0.70]$. At the end of the training, rats were divided into two groups that showed comparable levels of self-administration over the last two training sessions [i.e., reactivated propranolol treated (P) rats: $45 \pm 4.4$ rewards; reactivated saline (S) rats: $46 \pm 5.6$ rewards].

The effect of reactivation and treatment on behavior during the retention sessions was tested using a repeated measures analysis. This analysis revealed that three post-reactivation treatments with propranolol reduced alcohol seeking over all three retention days together as evidenced by a significant drug treatment effect

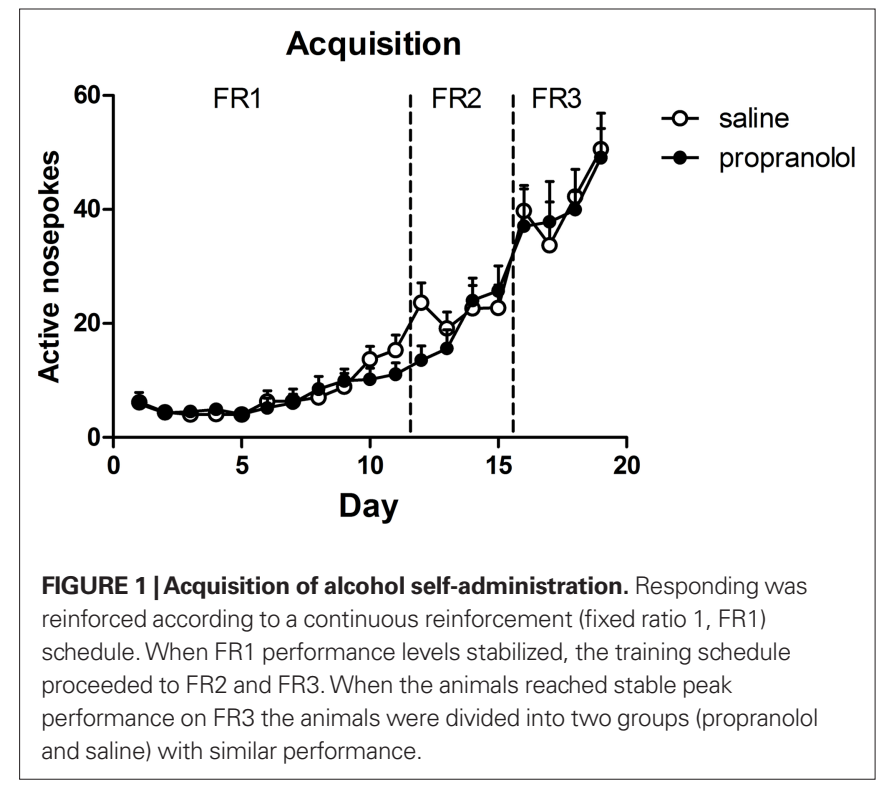

$[F(1,19)=7.067, p<0.05$; Figure 2A $]$. No significant within-subject interactions of retention session $\times$ drug treatment was observed $[F(2,38)=2.113, p>0.1]$.

A separate group of animals was trained for the no-reactivation condition. Similar to the animals that were reactivated the no-reactivation groups [i.e., no-reactivation propranolol (NP) and noreactivation saline (NS)] demonstrated equivalent levels of active responding during the last 2 days of the 19-day training period [no-reactivation: $F(1,15)<1, p>0.70$ ] and showed comparable levels of self-administration (NP: $43 \pm 2.1$ rewards; NS: $40.6 \pm 4.2$ rewards). In contrast, to what was seen in the reactivated condition repeated treatment without reactivation did not affect alcohol seeking $[F(1,15)<1, p>0.80$; Figure 2B $]$, indicating that alcohol seeking behavior was not affected by home cage injections of propranolol.

In all groups, we observed a significant main effect of retention session, meaning that responding decreased over repeated testing [reactivation: $F(2,38)=17.596, p<0.001$; no-reactivation: $F(2,30)=14.781, p<0.001]$.

\section{EXPERIMENT 2: POST-REACTIVATION MK801 (0.1 mg/kg)}

All groups were trained to stable peak performance and demonstrated equivalent levels of active responding during the last 2 days of training [reactivation: $F(1,14)<1, p>0.65$ ]. Reactivated MK801 treated $(\mathrm{MK})$ rats earned $43 \pm 5.3$ rewards and reactivated saline $(S)$ rats earned $41 \pm 2.5$ rewards over the last two training sessions. These levels of responding were comparable to responding in experiment 1 .

The repeated measures ANOVA just failed to show a significant between subject effect of treatment on behavior during the retention sessions $[F(1,14)=4.189, p=0.06$; Figure 3A $]$. This indicates that rats in the MK group had a strong tendency to show less alcohol seeking compared to the $S$ group in all three retention sessions.

A separate group of animals was trained to test the no-reactivation MK801 (NMK) and NS conditions. Similar to the reactivated group these animals demonstrated equivalent levels of active responding during the last 2 days of the 19-day training period [no-reactivation: $F(1,14)<1, p>0.50$ ] and earned comparable numbers of rewards 


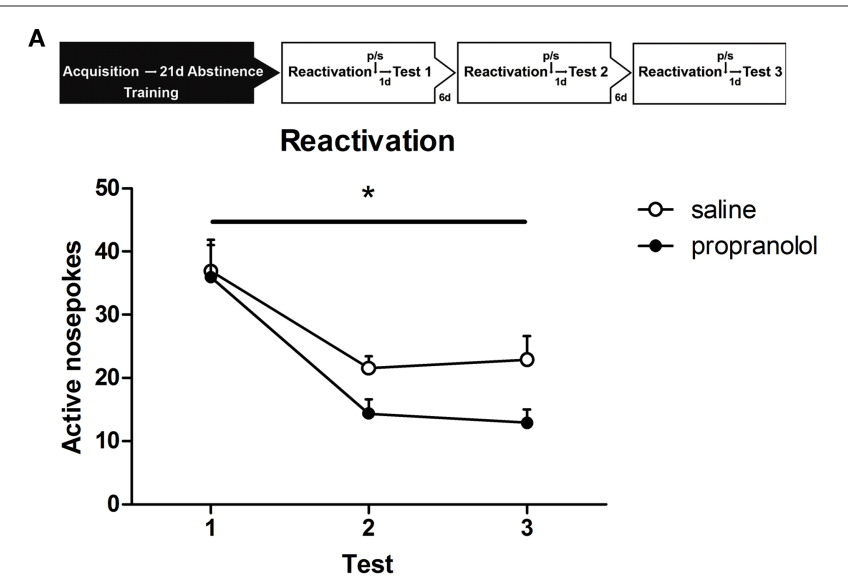

B

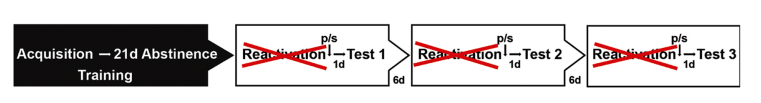

No - Reactivation

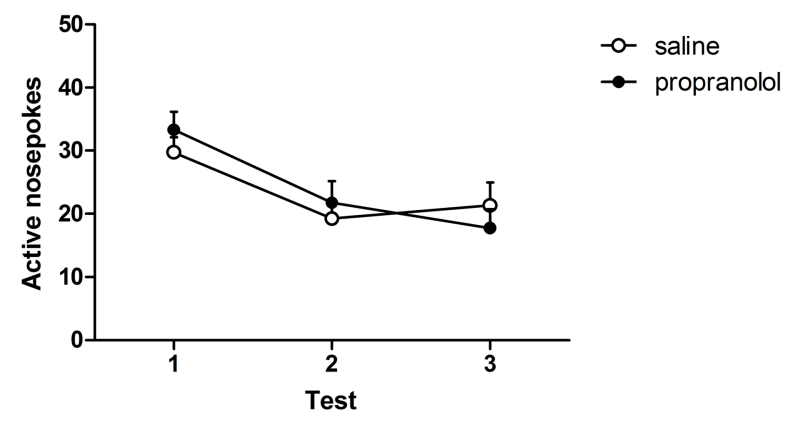

FIGURE 2 | Experimental setup. After 19 days of alcohol self-administration training, rats were left undisturbed for 21 days (abstinence). Subsequently, they were exposed to the self-administration context in which the house light and stimulus light were presented continuously and the compound cs was presented non-contingently (reactivation). Rats received a propranolol or saline injection directly after reactivation or received a propranolol or saline injection without reactivation (no-reactivation). One day later the animals were tested for memory retention (test). Reactivation and testing was repeated two times every 7 and 14 days after the first retrieval test. (A) Memory retention for alcohol self-administration after post-retrieval manipulation. Number of active nose pokes made during retention test 1, 2, and 3. (B) Memory retention for alcohol self-administration without reactivation. Number of active nose pokes made during retention test 1,2 , and 3 .

(NMK: $45 \pm 4.1$ and NS 40.6 \pm 4.2 rewards) during the last 2 days of training. Repeated treatment without reactivation did not affect alcohol seeking $[F(1,14)=1.256, p>0.25$; Figure 3B $]$, however, in contrast to the reactivated condition, NMK animals tended to increase responding compared to the NS group in all three retention sessions.

Again a significant main effect of retention session was observed, reduced responding was seen for all treatments [reactivation: $F(1.7,23)=14.197, p<0.001$; no-reactivation: $F(1,14)=9.234$, $p<0.01]$.

\section{DISCUSSION}

Using an operant alcohol self-administration model we demonstrate that (1) relatively old alcohol-related memories are prone to disruption in a reactivation-dependent manner and that (2) $\beta$-adrenoceptor mediated signaling is critically involved in reconsolidation of these memories. In addition, animals that received post-reactivation treatment with NMDA receptor antagonist MK801 showed a strong tendency to reduce alcohol seeking $(p=0.06)$. Moreover, we demonstrate that $(3)$ repeated post-reactivation treatment (repeated reactivation followed by propranolol injection), but not single treatment, can result in diminished relapse to alcohol seeking.

The current experiment adds to our previous findings on reconsolidation of appetitive memories (Diergaarde et al., 2006), demonstrating that memory reconsolidation of both sucroseand alcohol-related memories is dependent, at least in part, on $\beta$-adrenoceptor mediated signaling. Furthermore, these results complement and extend on recent findings from von der Goltz et al. (2009), who show that single post-reactivation treatment with protein syntheses inhibitor anisomycin reduces cue-induced alcohol seeking the following day and 7 days after treatment. Interestingly, this particular study reports a transient effect of post-reactivation MK801 treatment; alcohol seeking was reduced the following day, but not 7 days after treatment. It should be noted that this study used a saccharin-fading procedure during the alcohol acquisition phase. This complicates the interpretation of the results, as recent findings indicate that prior instrumental training for a natural rewarder influences reinstatement of drug seeking behavior (Clemens et al., 2010). In other words, environmental cues associated with the natural reinforcer may have interfered with the memory process. We have shown previously, that reconsolidation of appetitive (sucroserelated) memories is susceptible to interference (Diergaarde et al., 2006; Milton et al., 2008b), making it difficult to assess whether prior saccharin training may have interfered with the reconsolidation process in the von der Goltz study. However, our experiments, that did not involve sweetening to facilitate acquisition of alcohol selfadministration, suggest that alcohol-related memories are prone to disruption in a reactivation-dependent manner. It should be noted however, that in our study, the observed effect of MK-801 on memory reconsolidation was only modest. Although the effect of MK-801 treatment seems more apparent during the first test session than that of propranolol treatment, it did not quite reach significance over the three sessions. Thus, it seems that the long-term effects of post-reactivation manipulation of the NMDA receptor are less pronounced than the effects of protein synthesis inhibition or blockade of $\beta$-adrenoceptors. Recent studies by the Everitt group suggest that this might be due to a time-limited role of NMDA receptors in the reconsolidation process. Thus, in their hands, injections with the NMDA receptor antagonist APV or MK-801 were not effective in reducing instrumental responding for cocaine conditioned reinforcement when given post-retrieval but only when given prior to memory retrieval (Milton et al., 2008a). Similarly, MK-801 impaired conditioned sucrose seeking when it was administered before, but not after, a memory reactivation session (Lee and Everitt, 2008a,b; Milton et al., 2008a). Collectively, these studies, in accordance with data presented here, suggest that, in addition to the $\beta$-adrenoceptor, the NMDA receptor plays a (time-limited) role in reconsolidation of reward-related memories.

Our results are consistent with other studies suggesting that memories related to drugs of abuse are prone to disruption. A recent study addressing this issue in a cocaine self-administration 


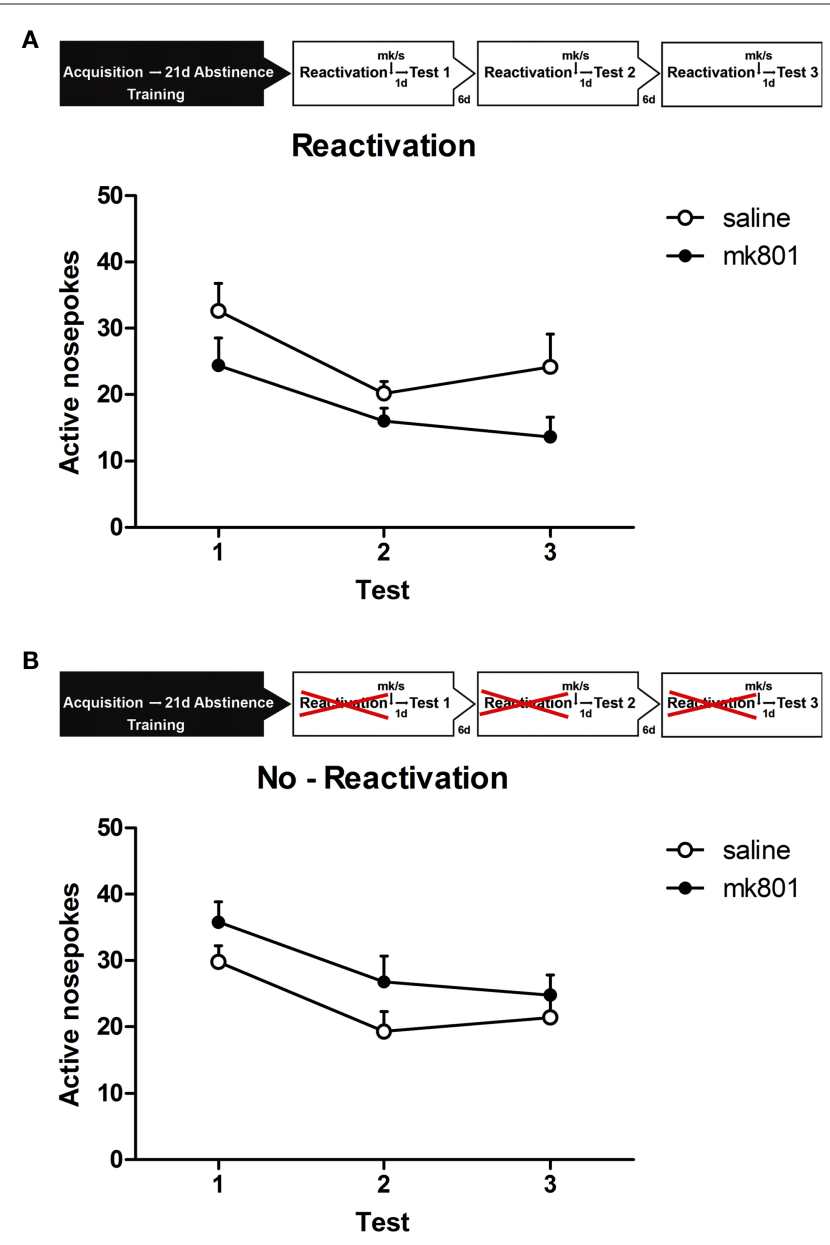

FIGURE 3 | Experimental setup. After 19 days of alcohol self-administration training, rats were left undisturbed for 21 days (abstinence). Subsequently, they were exposed to the self-administration context in which the house light and stimulus light were presented continuously and the compound cs was presented non-contingently (reactivation). Rats received a MK801 or saline injection directly after or received a MK801 or saline injection without reactivation (no-reactivation). One day later the animals were tested for memory retention (test). Reactivation and testing was repeated two times every 7 and 14 days after the first retrieval test. (A) Memory retention for alcohol self-administration after post-retrieval manipulation. Number of active nose pokes made during retention test 1,2, and 3. (B) Memory retention for alcohol self-administration without reactivation. Number of active nose pokes made during retention test 1,2 , and 3 .

paradigm demonstrated that pre-reactivation antagonism of NMDA receptor reduced the conditioned reinforcing properties of a previously cocaine-paired stimulus (Milton et al., 2008a). In addition several groups investigating drug-related reconsolidation in a conditioned place preference (CPP) paradigm have modulated place preference for cocaine, amphetamine, and morphine (Bernardi et al., 2006; Valjent et al., 2006; Robinson and Franklin, 2007; Sadler et al., 2007). However, a limitation of these studies is that CPP generally involves relatively new memories, use limited drug pairings and passive rather than active administration of drugs of abuse. In this respect using an reinstatement model designed to mimic aspects of alcohol addiction in humans more closely has several advantages. In particular it involves, (1) alcohol self-administration, (2) repeated exposure to alcohol and related stimuli, (3) alcohol-associated cues that can induce alcohol seeking and provoke relapse after abstinence, and (4) a 3-week post-training interval to model relapse after prolonged period of abstinence.

Alternative explanations for our findings need to be considered. Although retention tests were performed under drug free conditions, it is possible that non-specific or aversive properties of propranolol and MK801 resulted in the reduction of alcohol seeking that was observed. Given that amnestic effects in this experiment were reactivation-dependent, i.e., they were not found in the no-reactivation groups, non-associative effects of propranolol or MK801 do not explain our results. This notion is further substantiated by the fact that the doses of propranolol and MK801 used here have not been reported to induce aversive effects (Sara et al., 1995; Golden and Houpt, 2007; Milton et al., 2008b).

It might be argued that the observed reduction in alcohol seeking was caused by the fact that pharmacological treatments facilitated extinction rather than disrupting reconsolidation. This possibility, however, seems not very likely. In fact, several studies indicate that pharmacological activation rather than blockade of $\beta$-adrenergic or glutamatergic signaling facilitates (extinction) learning (Berman and Dudai, 2001; Bevilaqua et al., 2006; Popik et al., 2006; Gass and Olive, 2008; Vengeliene et al., 2008), whereas antagonism under certain circumstances impairs extinction learning (Bevilaqua et al., 2006; Hsu and Packard, 2008). Moreover, one of the characteristics of drug addiction is that extinguished drugrelated memories can reinstate spontaneously, due to drug priming, cue exposure, or stress. In contrast, no spontaneous recovery of responding for cocaine-associated cues was reported in a recent study that tested the persistence of memory impairment 15,22 , and 29 days after propranolol treatment (Milton et al., 2008b). Accordingly, cocaine primed reinstatement was abolished in animals that received repeated post-reactivation propranolol (FricksGleason and Marshall, 2008). Together, these observations suggest that a facilitated extinction could not explain our results.

In contrast to our findings, many groups investigating reconsolidation report that single treatment is sufficient to induce memory impairment in paradigms for fear conditioning, CPP, and operant instrumental learning (Debiec and LeDoux, 2004; Bernardi et al., 2006; Diergaarde et al., 2006; Milton et al., 2008b). This discrepancy may be explained by the nature of the memories that are involved in our experiment. Unlike fear-based learning tasks, that allow rapid association of negative emotionally laden stimuli, operant instrumental learning requires multiple training sessions, resulting in many cue-reward pairings. Additionally, our model involves a long post-training period. These methodological features are thought to strengthen memory formation and may reduce susceptibility for memory reconsolidation (Suzuki et al., 2004; Diergaarde et al., 2006). Moreover, the addictive nature of alcohol is an important factor influencing memory strength in our paradigm. In addiction, addictive substances, including alcohol, may employ neural systems that are normally involved in memory processes (Robbins et al., 2008). It is believed that drugs of abuse can induce aberrant stimulation of brain structures involved in reward learning, such as the ventral tegmental area, nucleus accumbens, and prefrontal cortex, leading to "overlearning” of drug-related cues (Hyman et al., 2006; Robbins et al., 2008). 
Alcohol is also known to affect processes that are associated with memory formation. For instance, alcohol exposure affects NMDAmediated synaptic plasticity and LTP expression (Hoffman and Tabakoff, 1996; Fadda and Rossetti, 1998; Hyman et al., 2006). These alcohol-related effects, together with the multiple training sessions and the age of the alcohol-related memories in our paradigm, may render memories more resistant to post-reactivation manipulations. Our approach of repeated treatment in order to affect these well established memories related to addictive substances is not unique. In fact some recent observations indicate that repeated disruption may be more effective than single. Using an amphetamine CPP paradigm Sadler et al. (2007) showed that only repeated treatment, consisting of memory activation tests followed by systemic MK-801 administration, reduced expression of a well established amphetamine-CPP. Differential effects of single and repeated post-retrieval systemic propranolol injections were also observed in a cocaine CPP procedure (Fricks-Gleason and Marshall, 2008). Although both single and repeated propranolol treatment resulted in a reduced place preference compared to the saline treated groups, repeated treatment but not single treatment abolished cocaine primed reinstatement. These results indicate that single propranolol treatment did weaken the memory but only repeated treatment disrupted memory for cocaine place preference. On the other hand, several studies, including the von der Goltz study, have observed effects of single treatment (Miller and Marshall, 2005; Bernardi et al., 2006; Diergaarde et al., 2006; Lee et al., 2006a; Valjent et al., 2006; Kelley et al., 2007; Robinson and Franklin, 2007; Itzhak, 2008; Wang et al., 2008; Zhai et al., 2008;

\section{REFERENCES}

Alberini, C. M. (2005). Mechanisms of memory stabilization: are consolidation and reconsolidation similar or distinct processes? Trends Neurosci. 28, 51-56.

Anton, R. F., O’Malley, S. S., Ciraulo, D. A., Cisler, R. A., Couper, D., Donovan, D. M., Gastfriend, D. R., Hosking, J. D., Johnson, B. A., LoCastro, J. S., Longabaugh, R., Mason, B. J., Mattson, M. E., Miller, W. R., Pettinati, H. M., Randall, C. L., Swift, R., Weiss, R. D., Williams, L. D., and Zweben,A. (2006). Combined pharmacotherapies and behavioral interventions for alcohol dependence: the COMBINE study: a randomized controlled trial. JAMA 295, 2003-2017.

Berman, D. E., and Dudai, Y. (2001). Memory extinction, learning anew, and learning the new: dissociations in the molecular machinery of learning in cortex. Science 291, 2417-2419.

Bernardi, R. E., Lattal, K. M., and Berger, S. P. (2006). Postretrieval propranolol disrupts a cocaine conditioned place preference. Neuroreport 17, 1443-1447.

Bevilaqua, L. R., Bonini, J. S., Rossato, J. I., Izquierdo, L. A., Cammarota, M., and Izquierdo, I. (2006). The entorhinal cortex plays a role in extinction. Neurobiol. Learn. Mem. 85, 192-197.

Chaudhri, N., Sahuque, L. L., and Janak, P. H. (2008). Context-induced relapse of conditioned behavioral responding to ethanol cues in rats. Biol. Psychiatry 64, 203-210.

Clemens, K. J., Caille, S., and Cador, M. (2010). The effects of response operandum and prior food training on intravenous nicotine self-administration in rats. Psychopharmacology (Berl.) 211, 43-54.

Debiec, J., and LeDoux, J. E. (2004). Disruption of reconsolidation but not consolidation of auditory fear conditioning by noradrenergic blockade in the amygdala. Neuroscience 129, 267-272.

De Vries, T. J., and Schoffelmeer, A. N. (2005). Cannabinoid CB1 receptors control conditioned drug seeking. Trends Pharmacol. Sci. 26, 420-426.

De Vries, T. J., Shaham, Y., Homberg, J. R., Crombag, H., Schuurman, K., Dieben, J., Vanderschuren, L. J., and Schoffelmeer, A. N., (2001). A cannabinoid mechanism in relapse to cocaine seeking. Nat. Med. 7, 1151-1154.

Diergaarde, L., Schoffelmeer, A. N., and De Vries, T. J. (2006). Beta-adrenoceptor mediated inhibition of long-term reward-related memory reconsolidation. Behav. Brain Res. 170, 333-336.

von der Goltz et al., 2009) even on reconsolidation of older drugrelated memories (Diergaarde et al., 2006; Lee et al., 2006b; von der Goltz et al., 2009). Variation in paradigms, such as species, training schedule, reactivation parameters, post-training interval, and extinction sessions, may explain these different observations. Indeed, successful modulation of reconsolidation appears very sensitive to the specific methodological parameters used (Sara, 2000; Tronson and Taylor, 2007). Nevertheless, collectively, these studies suggest that under certain conditions memories related to drug of abuse undergo reconsolidation. It is therefore of importance to elucidate the signaling pathways mediating NMDA receptor and $\beta$-adrenoceptor related effects on reconsolidation of drug of abuse related memories. The basolateral amygdala seems a likely candidate structure to study these molecular mechanisms, given that it is believed to be involved in associative reward learning and cue-induced relapse (Kalivas and McFarland, 2003; Kelley, 2004). Moreover, $\beta$-adrenoceptor and NMDA receptor antagonism in this region impairs reconsolidation of fear and cocaine self-administration related memories (Debiec and LeDoux, 2004; Milton et al., 2008a).

In conclusion we demonstrate in an animal model of relapse to alcohol seeking, that reconsolidation occurs for old alcoholrelated memories upon their reactivation and that particularly noradrenergic transmission plays an important role in this process. These findings suggest that pharmacological intervention in cue-exposure therapies for addictive behaviors may be useful in reducing relapse toward alcohol use and that repeated treatment could be beneficial.

Diergaarde, L., Schoffelmeer, A. N., and De Vries, T. J. (2008). Pharmacological manipulation of memory reconsolidation: towards a novel treatment of pathogenic memories. Eur. J. Pharmacol. 585, 453-457.

Fadda, F., and Rossetti, Z. L. (1998). Chronic ethanol consumption: from neuroadaptation to neurodegeneration. Prog. Neurobiol. 56, 385-431.

Fricks-Gleason, A. N., and Marshall, J. F. (2008). Post-retrieval beta-adrenergic receptor blockade: effects on extinction and reconsolidation of cocaine-cue memories. Learn. Mem. 15, 643-648.

Gass, J. T., and Olive, M. F. (2008). Positive allosteric modulation of mGluR5 receptors facilitates extinction of a cocaine contextual memory. Biol. Psychiatry 65, 717-720.

Golden, G. J., and Houpt, T. A. (2007) NMDA receptor in conditioned flavor-taste preference learning: blockade by MK- 801 and enhancement by D-cycloserine. Pharmacol. Biochem. Behav. 86, 587-596.

Hoffman, P. L., and Tabakoff, B. (1996) Alcohol dependence: a commentary on mechanisms. Alcohol Alcohol 31, 333-340.

Hsu, E., and Packard, M. G. (2008). Medial prefrontal cortex infusions of bupivacaine or AP-5 block extinction of amphetamine conditioned place preference. Neurobiol. Learn. Mem. 89, 504-512.

Hyman, S. E., Malenka, R. C., and Nestler, E. J. (2006). Neural mechanisms of addiction: the role of reward-related learning and memory. Annu. Rev. Neurosci. 29, 565-598.

Itzhak, Y. (2008). Role of the NMDA receptor and nitric oxide in memory reconsolidation of cocaine-induced conditioned place preference in mice. Ann. N. Y. Acad. Sci. 1139, 350-357.

Kalivas, P. W., and McFarland, K. (2003). Brain circuitry and the reinstatement of cocaine-seeking behavior. Psychopharmacology (Berl.) 168, 44-56.

Kelley, A. E. (2004). Ventral striatal control of appetitive motivation: role in ingestive behavior and reward-related learning. Neurosci. Biobehav. Rev. 27, 765-776.

Kelley, J. B., Anderson, K. L., and Itzhak, Y. (2007). Long-term memory of cocaine-associated context: disruption and reinstatement. Neuroreport 18, 777-780.

Lee, J. L., Di, C. P., Thomas, K. L., and Everitt, B. J. (2005). Disrupting reconsolidation of drug memories reduces 
cocaine-seeking behavior. Neuron 47 , 795-801.

Lee, J. L., and Everitt, B. J. (2008a). Appetitive memory reconsolidation depends upon NMDA receptor-mediated neurotransmission. Neurobiol. Learn. Mem. 90, 147-154.

Lee, J. L., and Everitt, B. J. (2008b). Reactivation-dependent amnesia in Pavlovian approach and instrumental transfer. Learn. Mem. 15, 597-602.

Lee, J. L., Milton, A. L., and Everitt, B. J. (2006a).Cue-induced cocaine seeking and relapse are reduced by disruption of drug memory reconsolidation. $J$. Neurosci. 26, 5881-5887.

Lee, J. L., Milton, A. L., and Everitt, B. J. (2006b). Reconsolidation and extinction of conditioned fear: inhibition and potentiation. J. Neurosci. 26, 10051-10056.

Miller, C. A., and Marshall, J. F. (2005). Molecular substrates for retrieval and reconsolidation of cocaine-associated contextual memory. Neuron $47,873-884$.

Milton, A. L., Lee, J. L., Butler, V. J., Gardner, R., and Everitt, B. J. (2008a). Intra-amygdala and systemic antagonism of NMDA receptors prevents the reconsolidation of drug-associated memory and impairs subsequently both novel and previously acquired drug-seeking behaviors. J. Neurosci. $28,8230-8237$.

Milton, A. L., Lee, J. L., and Everitt, B. J. (2008b). Reconsolidation of appetitive memories for both natural and drug reinforcement is dependent on $\{$ beta $\}$ adrenergic receptors. Learn. Mem. 15, 88-92.
Nader, K., Schafe, G. E., and Le Doux, J. E. (2000). Fear memories require protein synthesis in the amygdala for reconsolidation after retrieval. Nature 406, 722-726.

O'Brien, C.P.,Childress, A. R., Ehrman, R., and Robbins, S.J. (1998). Conditioning factors in drug abuse: can they explain compulsion? J. Psychopharmacol. 12, 15-22.

Popik,P.,Wrobel,M., and Bisaga,A. (2006). Reinstatement of morphine-conditioned reward is blocked by memantine. Neuropsychopharmacology 31, 160-170.

Przybyslawski, J., Roullet, P., and Sara, S. J. (1999). Attenuation of emotional and nonemotional memories after their reactivation: role of beta adrenergic receptors. J. Neurosci. 19, 6623-6628.

Przybyslawski, J., and Sara, S. J. (1997). Reconsolidation of memory after its reactivation. Behav. Brain Res. 84, 241-246.

Robbins, T. W., Ersche, K. D., and Everitt, B. J. (2008). Drug addiction and the memory systems of the brain. Ann. N. Y. Acad. Sci. 1141, 1-21.

Robinson, M. J., and Franklin, K. B. (2007). Central but not peripheral beta-adrenergic antagonism blocks reconsolidation for a morphine place preference. Behav. Brain Res. 182, 129-134.

Sadler, R., Herzig, V., and Schmidt, W. J. (2007). Repeated treatment with the NMDA antagonist MK-801 disrupts reconsolidation of memory for amphetamine-conditioned place preference. Behav. Pharmacol. 18, 699-703.
Sara, S. J. (2000). Retrieval and reconsolidation: toward a neurobiology of remembering. Learn. Mem. 7, 73-84.

Sara, S. J., Dyon-Laurent, C., and Herve, A. (1995). Novelty seeking behavior in the rat is dependent upon the integrity of the noradrenergic system. Brain Res. Cogn. Brain Res. 2, 181-187.

Suzuki, A., Josselyn, S.A., Frankland, P.W., Masushige, S., Silva, A. J., and Kida, S. (2004). Memory reconsolidation and extinction have distinct temporal and biochemical signatures. J. Neurosci. 24, 4787-4795.

Tronson, N. C., and Taylor, J. R. (2007) Molecular mechanisms of memory reconsolidation. Nat. Rev. Neurosci. 8, 262-275.

Valjent, E., Corbille, A. G., BertranGonzalez, J., Herve, D., and Girault, J. A. (2006). Inhibition of ERK pathway or protein synthesis during reexposure to drugs of abuse erases previously learned place preference. Proc. Natl. Acad. Sci. U.S.A. 103, 2932-2937.

Vengeliene, V., Kiefer, F., and Spanagel, R. (2008). D-cycloserine facilitates extinction of conditioned alcoholseeking behaviour in rats. Alcohol Alcohol. 43, 626-629.

von der Goltz, C., Vengeliene, V., Bilbao, A., Perreau-Lenz, S., Pawlak, C. R., Kiefer, F., and Spanagel, R. (2009). Cue-induced alcohol-seeking behaviour is reduced by disrupting the reconsolidation of alcohol-related memories. Psychopharmacology (Berl.) 205, 389-397.

Wang, X. Y., Zhao, M., Ghitza, U. E., Li, Y. Q., and Lu, L. (2008). Stress impairs reconsolidation of drug memory via glucocorticoid receptors in the basolateral amygdala. J. Neurosci. 28, 5602-5610.

WHO. (2004). WHO Global Status Report on Alcohol 2004. Geneva: WHO Department of Mental Health and Substance Abuse.

Zhai, H., Wu, P., Chen, S., Li, F., Liu, Y., and Lu, L. (2008). Effects of scopolamine and ketamine on reconsolidation of morphine conditioned place preference in rats. Behav. Pharmacol. 19, 211-216.

Conflict of Interest Statement: The authors declare that the research was conducted in the absence of any commercial or financial relationships that could be construed as a potential conflict of interest.

Received: 30 May 2010; paper pending published: 02 November 2010; accepted: 09 November 2010; published online: 26 November 2010.

Citation: Wouda JA, Diergaarde L, Riga $D$, van Mourik Y, Schoffelmeer ANM and De Vries TJ (2010) Disruption of longterm alcohol-related memory reconsolidation: role of $\beta$-adrenoceptors and NMDA receptors. Front. Behav. Neurosci. 4:179. doi: 10.3389/fnbeh.2010.00179

Copyright (C) 2010 Wouda, Diergaarde, Riga, van Mourik, Schoffelmeer and De Vries. This is an open-access article subject to an exclusive license agreement between the authors and the Frontiers Research Foundation, which permits unrestricted use, distribution, and reproduction in any medium, provided the original authors and source are credited. 